\title{
Editorial: Last Print Issue of Solar Physics
}

\author{
John Leibacher ${ }^{1,2,3}$ (D) Cristina H. Mandrini ${ }^{4}(D)$ \\ Lidia van Driel-Gesztelyi ${ }^{5,6,7}$ (D) \\ Michael S. Wheatland ${ }^{8}$
}

Published online: 14 December 2017

(c) Springer Science+Business Media B.V., part of Springer Nature 2017

This is the last print issue of Solar Physics!

This Editorial marks the end of print production of Solar Physics. At this point in time, probably very few readers would have noticed, so we thought that we should put it "in print" for the record. For many years now the electronic version of a Solar Physics article has been the "version of record", however we have maintained the print issue - until now. Starting with January 2018, volume 293, there will no longer be a print issue, after 51 years, 292 volumes, 640 issues, over 10,000 articles, 130,000 pages, and 8.5 meters on the bookshelf!

In addition, and independently of this change, Solar Physics transitioned to Continuous Article Publishing (CAP) at the beginning of 2017 with volume 292, to leave the restrictions

\footnotetext{
$凶$ J. Leibacher

john.leibacher.sola@gmail.com

C.H. Mandrini

mandrini@iafe.uba.ar

L. van Driel-Gesztelyi

lidia.vandriel@obspm.fr

M.S. Wheatland

michael.wheatland@sydney.edu.au

1 National Solar Observatory, Tucson, AZ, USA

2 Institut d'Astrophysique Spatiale - UMR-8617, Université Paris-Sud/CNRS, Orsay, France

3 Lunar and Planetary Laboratory, University of Arizona, Tucson, AZ, USA

4 Instituto de Astronomía y Física del Espacio, IAFE, Consejo Nacional de Investigaciones

Científicas y Técnicas, Universidad de Buenos Aires, Buenos Aires, Argentina

5 Observatoire de Paris, LESIA, CNRS, UPMC Université Paris-Diderot, Meudon, France

6 Mullard Space Science Laboratory, University College London, Dorking, UK

7 Konkoly Observatory, Hungarian Academy of Sciences, Budapest, Hungary

8 Sydney Institute for Astronomy, The University of Sydney, Sydney, NSW, Australia
} 
of the print world behind and to meet the demands and opportunities of the electronic world instead. With CAP, new issues are started online, with new articles appearing as soon as proofs are corrected. The sequence of articles is determined only by their order of appearance. The volumes no longer have a running page number, rather articles are identified by their volume and article number, and the page number for each article starts at one. Articles are uniquely identified by author name, journal title, volume, and article number, or simply the Digital Object Identifier (DOI).

Formerly we had occasional "Topical Issues", with articles accumulated online, and then all published as an issue. These have become "Topical Collections", with articles being assigned to an issue as any other article. The Topical Collection articles continue to have an identifying header and footer, including the names of the Guest Editors, on the first page. Topical Collections are prominently indicated as such in the contents of a volume, and they are accessible from a separate Topical Collections tab on the journal's content page.

Welcome to the new, paper-free Solar Physics - long live electronic articles!! 